\title{
Menarquia temprana y su asociación con conductas de riesgo en adolescentes
}

\author{
Ingrid Leal F. ${ }^{1 a}$, Valeria Stuardo A. $^{2 b}$, Temístocles Molina G. ${ }^{1 c}$, Electra González A. ${ }^{1 d}$ \\ ${ }^{1}$ Centro de Medicina Reproductiva y Desarrollo Integral del Adolescente (CEMERA), ${ }^{2}$ Escuela de Salud Pública. \\ Facultad de Medicina, Universidad de Chile.
}

${ }^{a}$ Matrona, MSc. ${ }^{b}$ Matrona, MSc, PhD. ${ }^{\text {C }}$ Bioestadístico, MSc. ${ }^{d}$ Asistente Social, MSc.

\section{RESUMEN}

Antecedentes: La pubertad no está necesariamente asociada a un desarrollo psicosocial equivalente. La falta de madurez y las características de la adolescencia podrían favorecer la aparición de conductas de riesgo. Objetivo: Determinar la asociación entre la edad de la menarquia y presencia de conductas de riesgo en adolescentes. Método: Diseño de corte transversal. La muestra incluyó adolescentes postmenárquicas. El instrumento fue una encuesta semiestructurada. Se realizó análisis descriptivo bivariado y multivariado. Resultados: La muestra correspondió a 1844 adolescentes, la mediana de edad fue de 16 años y 12 años para la menarquia. El $57 \%$ provino de nivel socioeconómico medio, el $98 \%$ estaba escolarizada y $52 \%$ vivía con ambos padres. El $35,1 \%$ presentó menarquia temprana. El abuso sexual se presentó en $26,0 \%$ en el grupo de menarquia temprana y en $21,4 \%$ en el de menarquia no temprana. El inicio de actividad sexual antes de los 15 años se presentó en un $39,4 \%$ y en $22,3 \%$ de adolescentes con menarquia temprana y no temprana respectivamente (OR: 1,88; IC95\%: 1,46-2,43), asociándose con disfuncionalidad familiar (OR: 2,08; IC95\%: 1,45-2,99) y ausencia de los padres (OR: 1,74; IC95\%: 1,09-2,76). La frecuencia de consumo de alcohol se asoció a disfuncionalidad familiar severa (OR: 2,00; IC95\%: 1,34-3,00). Conclusión: La menarquia temprana se asoció al inicio precoz de actividad sexual. Es importante promover comportamientos seguros desde edades tempranas y acompañar a las niñas en su proceso de maduración.

\section{PALABRAS CLAVES: Menarquia, adolescencia, conductas de riesgo}

\section{SUMMARY}

Background: Puberty is not necessarily associated with an equivalent psychosocial development. The lack of maturity and characteristics of adolescence may promote the emergence of risk behaviors. Objective: To determine the association between age at menarche and presence of risk behaviors in adolescents. Method: Cross-sectional design study. The sample included postmenarchal adolescents. The instrument was a semistructured survey. Bivariate and multivariate descriptive analysis. Results: The sample consisted of 1844 adolescents, the median age was 16 years and 12 years for menarche. $57 \%$ came from middle socioeconomic level, $98 \%$ attended school and $52 \%$ lived with both parents. $35.1 \%$ had early menarche. Sexual abuse occurred in $26.0 \%$ in the group of early menarche and $21.4 \%$ for no early menarche. The onset of sexual activity before age 15 occurred in $39.4 \%$ and $22.3 \%$ of adolescents with early and no early menarche respectively (OR: $1.88 ; 95 \% \mathrm{Cl}: 1.46-2.43$ ), associated with family dysfunction (OR: $2.08 ; 95 \% \mathrm{Cl}$ : 1.45-2.99) and absence of parents (OR: $1.74 ; 95 \% \mathrm{Cl}: 1.09-2.76$ ). The frequency of alcohol consumption was associated with severe family dysfunction (OR: $2.00 ; 95 \% \mathrm{Cl}: 1.34-3.00)$. Conclusion: Early menarche was associated with early onset of sexual activity. It is important to promote safe behavior from an early age and escort girls in their maturation process.

KEY WORDS: Menarche, adolescence, risk behaviors 


\section{INTRODUCCIÓN}

En Chile, la menarquia muestra desde el siglo XIX hasta la década de los setenta, una tendencia secular, similar a la de países desarrollados $(1,2,3)$. Las razones que la explicarían son: la reducción de las enfermedades, la mejor nutrición y el aumento de la obesidad infantil $(4,5)$.

Existen cambios cerebrales durante la pubertad que explicarían parte las conductas de riesgo. Por otro lado, la mayoría de los adolescentes atraviesan esta etapa sin experimentar dificultades $(6,7)$. Así las conductas riesgosas no se distribuyen aleatoriamente y se concentran en un subconjunto más vulnerable.

Dentro de las teorías que explicarían la asociación entre la edad de la menarquia y conductas de riesgo, está que el desarrollo puberal se presenta durante la adolescencia inicial (10 a 13 años), caracterizado por rápidos cambios físicos, lo que llevaría a una inadecuada autopercepción, asociado al impulso de experimentación y búsqueda de identidad, donde los pares toman gran importancia, habiendo un distanciamiento de la familia. Así, aquellas adolescentes que se desarrollan primero, presentan un retraso de la madurez psicosocial en relación a la física $(8,9)$. Otra explicación, a la luz de estudios sobre el desarrollo del cerebro y pubertad, sugieren que las habilidades cognitivas para hacer frente a roles sociales complejos, no se inician sincrónicamente con la pubertad, lo que generaría estrés para las adolescentes, aumentando los problemas de conducta (10). De acuerdo con esta perspectiva, las adolescentes son más vulnerables para las conductas de riesgo a principios de la adolescencia, ya que en esta etapa se acentúan las diferencias visibles entre las que se desarrollan primero y las que aún no han iniciado el proceso. A medida que las que se desarrollan primero se equiparan con las que se desarrollaron después, la relación entre la pubertad temprana y los problemas de comportamiento desaparecerían $(11,9)$. Se ha asociado la menarquia temprana a variables como: relaciones sexuales precoces, embarazos adolescentes, infecciones de transmisión sexual, episodios de embriaguez y uso de tabaco y drogas $(3,9,12,13,14)$. Los efectos a largo plazo del inicio temprano de la menarquia pueden relacionarse con áreas del desarrollo con consecuencias hasta la adultez.

El objetivo de este estudio fue determinar la asociación entre la edad de la menarquia y presencia de conductas de riesgo en adolescentes consultantes en el Centro de Medicina Reproductiva y Desarrollo Integral del Adolescente (CEMERA) entre los años 2005 a 2010. Se trabajó con el concepto de menarquia temprana ( $\leq 11$ años).

\section{PACIENTES Y MÉTODOS}

Diseño de corte transversal. La muestra correspondió a adolescentes entre 12 a 19 años postmenárquicas, consultantes en el CEMERA entre los años 2005 y 2010. El instrumento de recolección correspondió a una encuesta semi-estructurada. Los datos fueron ingresados en base de datos.

La variable edad de menarquia se trabajó de forma continua para estimar, en promedio, la edad de inicio de la conducta de riesgo través de la prueba de $t$ de Student. Luego se dicotomizó en temprana y no temprana. Las variables dependientes fueron: inicio sexual precoz (antes o después de los 15 años), número de parejas sexuales (número de parejas distintas con las que ha mantenido relaciones sexuales coitales), consumo y frecuencia de uso de tabaco (consumo diario o semanal y menos), consumo y frecuencia de uso de alcohol (2 veces a la semana o más y semanal o menos). Las variables sociodemográficas correspondieron a: nivel socio-económico (clasificación de acuerdo al esquema de Graffar), escolaridad (último curso escolar completo), dinámica familiar (puntaje Apgar), estructura familiar (presencia en el hogar de ambos padres, uno de sus padres o ninguno de ellos), antecedente abuso sexual (auto reporte). La comparación de proporciones se realizó según prueba de ji cuadrado. La medida de fuerza de asociación fue evaluada por Odds Ratio a través de regresión logística univariada y multivariada. Se consideraron valores $p<0,05$, con sus intervalos de confianza. Se controló por variables confusoras.

\section{RESULTADOS}

La muestra correspondió a 1844 adolescentes, la mediana y el promedio de edad fue de 16 años (rango: 12 - 19 años), la mediana de la menarquia fue de 12 años (rango: 9 a 15 años) y el promedio 12,07 años (IC: 12,00 - 12,12) con una desviación estándar de 0,03 años. El 35,08\% presentó menarquia temprana ( $\leq 11$ años). La distribución de factores sociodemográficos fue similar en ambos grupos. El antecedente de abuso sexual se presentó en un $25,47 \%$ en el grupo de menarquia temprana y en un $21,42 \%$ en las adolescentes de menarquia no temprana. Los resultados del análisis descriptivo se resumen en la Tabla I.

En cuanto a las conductas de riesgo, la mediana de edad de inicio de consumo de tabaco, alcohol y drogas fue menor en las adolescentes con menarquia temprana. La mediana para inicio de relaciones sexuales fue de 15 años para ambos grupos. La descripción de las conductas de riesgo se resume en la Tabla II. 
Tabla I

DESCRIPCIÓN DE LA MUESTRA SEGÚN COVARIABLES

\begin{tabular}{|c|c|c|c|}
\hline Variable & $\begin{array}{l}\text { Menarquia temprana } \\
\text { (n: 647) }\end{array}$ & $\begin{array}{l}\text { Menarquia no temprana } \\
\text { (n: 1197) }\end{array}$ & $\begin{array}{c}\text { Total adolescentes } \\
\text { (n: 1844) }\end{array}$ \\
\hline Edad a la encuesta (media) & 15,58 años & 16,02 años & 16 años \\
\hline \multicolumn{4}{|l|}{ Nivel socio-económico: } \\
\hline Alto & $29(4,75 \%)$ & $45(3,97 \%)$ & $74(4,24 \%)$ \\
\hline Medio & $348(57,05 \%)$ & $657(57,94 \%)$ & $1005(57,63 \%)$ \\
\hline Bajo & $233(38,20 \%)$ & $432(38,10 \%)$ & $665(38,13 \%)$ \\
\hline \multicolumn{4}{|l|}{ Escolaridad: } \\
\hline Básica & $95(14,71 \%)$ & $87(7,29 \%)$ & $182(9,89 \%)$ \\
\hline Media & $551(85,29 \%)$ & $1106(92,63 \%)$ & $1657(90,05 \%)$ \\
\hline \multicolumn{4}{|l|}{ Dinámica familiar: } \\
\hline Funcional & $404(62,54 \%)$ & $715(60,03 \%)$ & $1119(60,91 \%)$ \\
\hline Disfunción moderada & $161(24,92 \%)$ & $323(27,12 \%)$ & $484(26,35 \%)$ \\
\hline Disfunción severa & $81(12,54 \%)$ & $153(12,85 \%)$ & $234(12,74 \%)$ \\
\hline \multicolumn{4}{|l|}{ Estructura de familia: } \\
\hline Biparental & $344(53,17 \%)$ & $624(52,17 \%)$ & $968(52,52 \%)$ \\
\hline Monoparental & $266(40,19 \%)$ & $467(39,05 \%)$ & $727(39,45 \%)$ \\
\hline Otra tipo de familia & $43(6,65 \%)$ & $105(8,78 \%)$ & $148(8,03 \%)$ \\
\hline Antecedentes de abuso sexual & $162(25,47 \%)$ & $251(21,42 \%)$ & $413(22,84 \%)$ \\
\hline
\end{tabular}

\section{Tabla II}

DESCRIPCIÓN DE LA MUESTRA SEGÚN VARIABLES CONDUCTUALES

\begin{tabular}{lccc}
\hline Variable & $\begin{array}{c}\text { Menarquia temprana } \\
\text { (n: } 647)\end{array}$ & $\begin{array}{c}\text { Menarquia no temprana } \\
\text { (n: } 1197)\end{array}$ & $\begin{array}{c}\text { Total adolescentes } \\
\text { (n: } 1844)\end{array}$ \\
\hline Consumo de tabaco & $376(64,16 \%)$ & $717(64,42 \%)$ & $1093(64,33 \%)$ \\
Consumo de alcohol & $372(63,48 \%)$ & $746(67,09 \%)$ & $1118(65,84 \%)$ \\
Consumo de drogas & $204(35,29 \%)$ & $377(34,06 \%)$ & $581(34,48 \%)$ \\
Edad inicio consumo de tabaco (media) & 13 años & 14 años & 14 años \\
Edad inicio consumo de alcohol (media) & 14 años & 15 años & 15 años \\
Inicio actividad sexual & $528(83,02 \%)$ & $953(79,95 \%)$ & $1477(81,02 \%)$ \\
Edad inicio actividad sexual (media) & 15 años & 15 años & 15 años \\
Actividad sexual precoz (<15 años) & $208(39,39 \%)$ & $213(22,34 \%)$ & $421(28,43 \%)$ \\
Una pareja sexual & $377(71,54 \%)$ & $682(71,79 \%)$ & $1059(71,70 \%)$ \\
Dos parejas sexuales & $114(21,63 \%)$ & $182(19,16 \%)$ & $296(20.04 \%)$ \\
Tres o más parejas sexuales & $36(6,83 \%)$ & $86(9,05 \%)$ & $122(15,88 \%)$ \\
\hline
\end{tabular}

La Tabla III muestra el análisis de comparación entre los promedios de edad de inicio de cada conducta de riesgo, según grupo de menarquia. Para este análisis se utilizó T de Student. Se observa un promedio de edad de inicio de conductas de riesgo, levemente menor en las adolescentes con menarquia temprana, siendo esta diferencia estadísticamente significativa en las tres conductas $(p=0,005)$. 
Tabla III

PROMEDIO DE EDAD DE INICIO DE CONDUCTAS DE RIESGO SEGÚN EDAD DE LA MENARQUIA

\begin{tabular}{lccc}
\hline Variable & $\begin{array}{c}\text { Menarquia temprana } \\
\text { (n: } 647) \\
\text { Años }\end{array}$ & $\begin{array}{c}\text { Menarquia no temprana } \\
\text { (n: 1197) } \\
\text { Años }\end{array}$ & $\begin{array}{c}\text { Total adolescentes } \\
\text { (n: 1844) } \\
\text { Valor } p\end{array}$ \\
\hline Consumo de tabaco & 13,33 & 13,90 & 0,000 \\
Consumo de alcohol & 14,06 & 14,61 & 0,000 \\
Consumo de drogas & 14,25 & 14,66 & 0,025 \\
Actividad sexual & 14,83 & 15,34 & 0,000 \\
\hline
\end{tabular}

En el análisis bivariado se observa que la mayoría de las adolescentes han iniciado actividad sexual $(81,01 \%)$, no encontrándose diferencias de acuerdo a la edad de la menarquia $(p=0,111)$. En cuanto al número de parejas sexuales, no se encontró diferencia de acuerdo a la edad de la menarquia. Se observó mayor frecuencia de tres o más parejas sexuales en adolescentes con antecedente de abuso sexual $(p=0,002)$.

No se observó diferencias significativas entre edad de la menarquia y consumo de tabaco $(p=0,916)$. No hubo asociación entre frecuencia de consumo de tabaco y edad de la menarquia.

Para el consumo de alcohol no se encontró diferencias significativas de acuerdo a la edad de la menarquia. La frecuencia de consumo de alcohol fue significativamente más alta en adolescentes con disfunción familiar severa y antecedente de abuso sexual (Tabla IV).
Para cada conducta de riesgo, se realizaron modelos de regresión por separado. Así al ajustar por todas las covariables, el factor de riesgo edad de la menarquia temprana, aumenta la probabilidad de tener sexo precoz en 1,88 veces ( $88 \%$ más). También resultan significativas las covariables: Edad, es decir, por cada año de aumento en la edad, el riesgo de iniciar actividad sexual precozmente disminuye un 56,7\%, En adolescentes con escolaridad básica el riesgo de iniciar actividad sexual precozmente disminuye un 40,4\%. En adolescentes con disfunción familiar severa el riesgo de iniciar actividad sexual precozmente aumenta un 108\%. En adolescentes con estructura familiar diferente a la bi o mono parental, el riesgo de iniciar actividad sexual precozmente aumenta un $74 \%$ (Tabla V).

Tabla IV

RESUMEN ANÁLISIS BIVARIADO DE LAS CONDUCTAS DE RIESGO

\begin{tabular}{lccccccc}
\hline Covariables & $\begin{array}{c}\text { Inicio } \\
\text { actividad } \\
\text { sexual }\end{array}$ & $\begin{array}{c}\text { Actividad } \\
\text { sexual } \\
\text { precoz }\end{array}$ & $\begin{array}{c}\text { Número } \\
\text { parejas } \\
\text { sexuales }\end{array}$ & $\begin{array}{c}\text { Consumo } \\
\text { de tabaco }\end{array}$ & $\begin{array}{c}\text { Consumo } \\
\text { de alcohol }\end{array}$ & $\begin{array}{c}\text { Frecuencia } \\
\text { consumo } \\
\text { tabaco }\end{array}$ & $\begin{array}{c}\text { Frecuencia } \\
\text { consumo } \\
\text { alcohol }\end{array}$ \\
\hline $\begin{array}{l}\text { Edad de la menarquia } \\
\text { temprana }\end{array}$ & 0,111 & 0,000 & 0,216 & 0,916 & 0,136 & 0,560 & 0,604 \\
$\begin{array}{l}\text { Nivel socio-económico } \\
\text { bajo }\end{array}$ & 0,611 & 0,055 & 0,580 & 0,356 & 0,079 & 0,356 & 0,057 \\
$\begin{array}{l}\text { Disfunción familiar } \\
\text { severa }\end{array}$ & 0,003 & 0,009 & 0,061 & 0,000 & 0,001 & 0,768 & 0,006 \\
$\begin{array}{l}\text { Estructura familiar sin } \\
\text { padres }\end{array}$ & 0,959 & 0,002 & 0,101 & 0,278 & 0,628 & 0,574 & 0,230 \\
\begin{tabular}{l} 
Abuso sexual \\
\hline
\end{tabular} & 0,011 & 0,054 & 0,002 & 0,005 & 0,009 & 0,613 & 0,023 \\
\hline
\end{tabular}




\section{INICIO DE ACTIVIDAD SEXUAL PRECOZ. ANÁLISIS DE REGRESIÓN LOGÍSTICA}

\begin{tabular}{lccccc}
\hline Variables & $\begin{array}{c}\text { Odds } \\
\text { Ratio }\end{array}$ & $\begin{array}{c}\text { Error } \\
\text { estándar }\end{array}$ & Valor Z & Valor $\mathrm{p}$ & $\begin{array}{c}\text { Intervalo de } \\
\text { confianza }\end{array}$ \\
\hline Edad menarquia temprana & 1,88 & 0,24 & 4,86 & 0,000 & $1,46-2,43$ \\
Edad & 0,43 & 0,02 & $-12,51$ & 0,000 & $0,38-0,49$ \\
NSE medio & 1,08 & 0,35 & 0,25 & 0,803 & $0,56-2,06$ \\
NSE bajo & 1,26 & 0,42 & 0,71 & 0,477 & $0,65-2,43$ \\
Escolaridad & 0,59 & 0,13 & $-2,22$ & 0,027 & $0,37-0,94$ \\
Disfunción familiar moderada & 1,04 & 0,16 & 0,32 & 0,751 & $0,77-1,41$ \\
Disfunción familiar severa & 2,08 & 0,38 & 3,97 & 0,000 & $1,45-2,99$ \\
Familia monoparental & 1,20 & 0,16 & 1,39 & 0,164 & $0,92-1,58$ \\
Otra familia & 1,74 & 0,41 & 2,36 & 0,018 & $1,09-2,76$ \\
Abuso sexual & 1,74 & 0,19 & 1,63 & 0,104 & $0,95-1,70$
\end{tabular}

NSE: nivel socioeconómico. Valor $p$ del modelo $=0,000$

En cuanto al consumo de tabaco y alcohol, la variable edad de la menarquia no fue significativa $(p=0,162$ y $p=0,661$ respectivamente). Sin embargo, resultó significativa la covariable edad, así por cada año que aumenta la edad la probabilidad de tener un consumo frecuente de tabaco es de $19 \%$, ajustado por todas las covariables. Las variables edad, disfunción familiar severa y abuso sexual, resultaron significativas como factores de riesgo para el consumo frecuente de alcohol, aumentando esta probabilidad en un $30 \%, 200 \%$ y $50 \%$ respectivamente.

\section{DISCUSIÓN}

La asociación entre la edad de la menarquia y el uso de sustancias e inicio de actividad sexual ha sido atribuida a factores biológicos, psicológicos y sociales. Es ampliamente reconocido que la pubertad tiene una naturaleza multidimensional, siendo un proceso no lineal, con ritmos cambiantes. Ellis y cols postulan que las niñas tienen condiciones para adaptarse, detectar y codificar la información sobre los niveles de apoyo y adversidad de su entorno en etapas tempranas, calibrando la magnitud y la activación de respuesta a distintos estresores, los cuales podrían adelantar el desarrollo puberal como atrasarlo, dependiendo del tipo de estresor y el momento en que se presente (15).

La mediana de edad de la menarquia, así como una proporción de adolescentes con menarquia temprana es similar a la reportada en otros estudios $(35,08 \%)(4,3,16,17)$. Esta disminución de la edad de la menarquia también podría explicar la menor variabilidad observada en cuanto a su distribución.
La distribución de variables sociodemográficas fue similar en ambos grupos. La frecuencia de familias con alto grado de disfuncionalidad y con estructura, es diferente a la biparental, y fueron antecedentes relevantes al estar señalados dentro de la literatura como estresores y relacionados con el inicio temprano de la pubertad y conductas de riesgo (18).

Es importante el alto porcentaje de abusos sexuales reportados, llegando al $22,8 \%$ y siendo ligeramente mayor en las adolescentes con menarquia temprana. Este antecedente ha sido fuertemente asociado a menarquia temprana. Aunque no está claro el mecanismo, sobre todo porque los estudios no han podido determinar la temporalidad de los eventos de esta asociación $(19,20,21)$. En el análisis bivariado se encontró asociación entre abuso sexual e inicio precoz de la actividad sexual, número de parejas sexuales, consumo de tabaco y frecuencia de consumo de alcohol, lo cual pone al antecedente de abuso sexual como una de las variables que se asocia más frecuentemente con alguna conducta de riesgo.

Las conductas de riesgo estudiadas, presentaron en esta población una proporción similar a lo descrito a nivel nacional, siendo algo diferente la conducta sexual. Esto último se relacionaría, con las características del centro de dónde provino la población estudiada, cuyo enfoque es la salud sexual y reproductiva, representando a una población específica y más vulnerable dentro de la población adolescente total.

El análisis de promedios de edad de inicio de las conductas de riesgo mostró una pequeña diferencia, siendo relevante desde un punto de vista clínico (21). En el análisis bivariado se encontró 
asociación entre la edad de la menarquia y el inicio precoz de la actividad sexual estos resultados coinciden con diversos estudios a nivel internacional $(8,3,13,14)$.

También resultaron ser factores de riesgo del inicio precoz de la actividad sexual, el provenir de una familia con disfunción severa y tener una estructura familiar en la cual no estén presentes ninguno de los padres. Estos hallazgos son plausibles, conociendo que la dinámica familiar, como la ausencia de los padres pueden ser estresores relevantes actuando como aceleradores de la pubertad (15).

Los datos señalan que el inicio de las relaciones sexuales es posterior a la menarquia, lo que nos da indicios de la direccionalidad de esta asociación. Para otras variables de la conducta sexual, como número de parejas sexuales, no se encontró diferencia, según edad de la menarquia. Sin embargo, si se encontró asociación entre el número de parejas sexuales y el antecedente de abuso sexual, siendo por lo tanto esta variable, probablemente, más determinante en el número de parejas sexuales que la edad de la menarquia. Cabe destacar que el inicio precoz de las relaciones sexuales conlleva riesgo de embarazos no deseados e infecciones de transmisión sexual, siendo el primero uno de los problemas de salud pública más relevantes, especialmente preocupante es el aumento de los embarazos en las adolescentes menores de 15 años (22).

En este estudio no se encontró asociación entre la edad de la menarquia y conductas de consumo y/o frecuencia de consumo de tabaco o alcohol, a diferencia de lo encontrado en otras investigaciones $(10,12,23,25)$. Sin embargo, coinciden estos resultados con otras investigaciones, donde tampoco se encontró asociación, o la medida de asociación referida incluyó el valor de nulidad $(3,24)$. Es posible que la población del presente estudio, siendo más homogénea en aspectos socioculturales, no haya revelado la posible asociación de las conductas de riesgo con la edad de la menarquia, o bien esta variable toma más relevancia en otras culturas y sociedades que la estudiada aquí. Otra posible explicación para no haber encontrado asociación, sería que el efecto de la precocidad de la edad de la menarquia sobre las conductas de riesgo, que se disipa a medida que se avanza en la edad. Al incluirse dentro de la población, adolescentes en distintas etapas, podría haber sesgado la asociación. Cabe destacar la asociación entre consumo frecuente de alcohol y disfunción familiar severa, $100 \%$ más de riesgo versus familias funcionales, y abuso sexual $50 \%$ más de riesgo versus adolescentes sin este antecedente. Estas asociaciones han sido descritas en otros estudios, demostrando la influencia del componente social y familiar en la conducta de los y las adolescentes $(3,18)$.
Dentro de las limitaciones de este estudio se encuentra que la población de estudio corresponde a una población específica consultante. Sin embargo, representa a una población de relativo difícil acceso. Por otro lado, la información utilizada fue recogida desde una fuente secundaria, encontrándose registros incompletos, principalmente en lo referente a consumo y frecuencia de uso de drogas, razón por la cual no se pudo utilizar esta información para el análisis, que se había planteado en un comienzo. Por otro lado, una fortaleza de este estudio es ser uno de los primeros estudios en Chile que investiga la relación de la menarquia con conductas de riesgo, entregando una primera impresión de lo que ocurre con la asociación de estas variables.

Es importante tener presente que la morbilidad y mortalidad en el grupo de adolescentes se relaciona con las conductas de riesgo, las que de mantenerse pueden llegar a la adultez. Esto pone de manifiesto la necesidad de realizar actividades de promoción y prevención de salud a edades más tempranas, así como acompañar a las niñas en su proceso de desarrollo durante la adolescencia, incorporando en los curriculum de formación en las escuelas, programas de educación sobre el papel de la pubertad en la salud, promoviendo el desarrollo de habilidades sociales para un mejor afrontamiento y transición de la etapa de la adolescencia. También es relevante la promoción de la educación sexual, que actualmente en nuestro país se imparte a contar de la enseñanza media, que a la luz de los resultados de este estudio, se debería iniciar durante los primeros ciclos de la enseñanza básica.

\section{CONCLUSIÓN}

Este estudio se suma a la evidencia que muestra la asociación entre la edad de la menarquia y ciertas conductas de riesgo, en particular el inicio precoz de las relaciones sexuales, la cual se asocia a importantes problemas de salud publica en Chile, como es el embarazo adolescente y las infecciones de transmisión sexual. Se propone continuar con estudios prospectivos que ayuden a aclarar el papel que juega la temporalidad del inicio de la pubertad en eventos sociales y psicológicos durante la etapa de la adolescencia.

\section{REFERENCIAS}

1. Zainab A, Babay C. Age at menarche and the reproductive performance of Saudi women. Ann Saudi Med 2004;24(5):354-6.

2. Rokade SA, Mane AK. A study of age at menarche, the secular trend and factors associated with it. The Internet Journal of Biological Anthropology 2008;3(2). Disponible en: https://ispub.com/lJBA/3/2/7469.

3. Gaudineau A, Ehlinger V, Vayssiere C, Jouret B, 
Arnaud C, Godeau E. Factors associated with early menarche: results from the French health behaviour in school-aged children (HBSC) study. BMC Public Health 2010;10:175.

4. Hernández MI, Unanue N, Gaete X, Cassorla F, Codner E. Edad de la menarquia y su relación con el nivel socioeconómico e índice de masa corporal. Rev Méd Chile 2007;135:1429-36.

5. Golub MS, Collman GW, Foster PM, Kimmel CA, Rajpert-De Meyts E, Reiter EO, Sharpe RM, Skakkebaek NE, Toppari J. Public health implications of altered puberty timing. Pediatrics 2008;121 Suppl 3:S218-30.

6. Oliva A. Desarrollo cerebral y asunción de riesgos durante la adolescencia. Apuntes de Psicología 2007;25:239-54.

7. Committee on the Science of Adolescence; Board on Children, Youth and Families; Institute of Medicine and National Research Council. THE Science of Adolescent Risk-Taking: Workshop Report. Washington, DC: 2011. Disponible en: http://www.nap.edu/catalog/12961.html

8. Downing J, Bellis MA. Early pubertal onset and its relationship with sexual risk taking, substance use and anti-social behaviour: a preliminary cross-sectional study. BMC Public Health 2009;9:446.

9. Tanner-Smith EE. Negotiating the early developing body: pubertal timing, body weight, and adolescent girls' substance use. J Youth Adolesc 2010;39:140216.

10. Kaltiala-Heino R, Koivisto A-M, Marttunen M, Fröjd S. Pubertal timing and substance use in middle adolescence: a 2-year follow-up study. J Youth Adolesc 2011;40:1288-301.

11. Jessor R. Risk behavior in adolescence: A psychosocial framework for understanding and action. J Adolesc Health 1991;12:597-605.

12. Lanza ST, Collins LM. Pubertal timing and the onset of substance use in females during early adolescence. Prev Sci 2002;3:69-82.

13. Glynn JR, Kayuni N, Floyd S, Banda E, FrancisChizororo M, Tanton $\mathrm{C}$, et al. Age at menarche, schooling, and sexual debut in northern Malawi. PloS One 2010;5(12):e15334.

14. Boden JM, Fergusson DM, Horwood LJ. Age of menarche and psychosocial outcomes in a New Zealand birth cohort. J Am Acad Child Adolesc Psychiatry 2011;50(2):132-40.

15. Ellis BJ, Shirtcliff EA, Boyce WT, Deardorff J, Essex MJ. Quality of early family relationships and the timing and tempo of puberty: effects depend on biological sensitivity to context. Dev Psychopathol 2011;23(1):85-99.

16. Al-Sahab B, Ardern Cl, Hamadeh MJ, Tamim H. Age at menarche in Canada: results from the National Longitudinal Survey of Children \& Youth. BMC Public Health 2010;10(1):736.

17. Anderson SE, Must A. Interpreting the continued decline in the average age at menarche: results from two nationally representative surveys of U.S. girls studied 10 years apart. J Pediatr 2005;147(6):753-60.

18. Jean RT, Wilkinson AV, Spitz MR, Prokhorov A, Bondy M, Forman MR. Psychosocial risk and correlates of early menarche in mexican-american girls. Am J Epidemiol 2011;173(10):1203-10.

19. Boynton-Jarrett R, Harville EW. A prospective study of childhood social hardships and age at menarche. Ann Epidemiol 2012;22(10):731-7.

20. Boynton-Jarrett R, Wright RJ, Putnam FW, Lividoti Hibert E, Michels KB, Forman MR, et al. Childhood abuse and age at menarche. J Adolesc Health 2013;52(2):241-7.

21. Henrichs K., McCauley $\mathrm{H}_{\text {., }}$ et al.: Early menarche and childhood adversities in a nationally representative sample. Int J Pediatr Endocrinol 2014;2014(1):14.

22. Gómez PI, Molina R, Zamberlin N. Factores relacionados con el embarazo y la maternidad en menores de 15 años en América Latina y el Caribe. FLASOG. Luis Tavara L, editor. Medicina. Lima; 2011.

23. González EM, Montero A, Martínez V. Comportamientos sexuales y diferencias de género en adolescentes usuarios de un sistema público de salud universitario. Rev Méd Chile 2007;135:1261-9.

24. Al-Sahab B, Ardern Cl, Hamadeh MJ, Tamim H. Age at menarche and current substance use among $\mathrm{Ca}-$ nadian adolescent girls: results of a cross-sectional study. BMC Public Health 2012;12(1):195.

25. Lien L, Haavet OR, Dalgard F. Do mental health and behavioural problems of early menarche persist into late adolescence? A three year follow-up study among adolescent girls in Oslo, Norway. Soc Sci Med 2010;71(3):529-33. 